\title{
Insecticide susceptibility and dengue vector status of wild Stegomyia albopicta in a strategically important area of Assam, India
}

\author{
Sunil Dhiman*, Bipul Rabha, Kavita Yadav, Indra Baruah and Vijay veer
}

\begin{abstract}
Background: Dengue vector control programmes are facing operational challenges due to resistance against commonly used insecticides throughout the endemic countries. Recently, there has been appreciable increase in the dengue cases in India, however, no recent data are available on susceptible status of dengue vectors. We have studied the susceptibility level of St. albopicta to commonly used insecticides in India. Adult mosquitoes were tested for the presence of dengue virus.

Methods: St. albopicta larval bioassays were carried out to determine the lethal concentrations $\left(\mathrm{LC}_{10}, \mathrm{LC}_{50}\right.$ and $\left.L C_{99}\right)$ and the resistance ratios ( $R_{10}, R_{50}$ and $\left.R R_{99}\right)$ for temephos. Susceptibility to $4 \% \mathrm{DDT}, 0.05 \%$ deltamethrin and $5 \%$ malathion was assessed following standard procedure. Knock-down times (KDT $10, \mathrm{KDT}_{50}$ and KDT 99 ) were estimated and knock-down resistance ratios $\left(\mathrm{KRR}_{10}, \mathrm{KRR}_{50}\right.$ and $\left.\mathrm{KRR}_{99}\right)$ were calculated. VectorTest ${ }^{\mathrm{TM}}$ dengue antigen assay was used to detect the dengue virus in the field collected mosquitoes.

Results: In larval bioassays, the RR ranged from 1.4 (for RRg9) to 1.7 (for $R_{50}$ ), which suggested that the tested St. albopicta were susceptible to temephos. There was no deviation among the lethal concentration data from linearity $\left(r^{2}=0.61\right)$. Adult St. albopicta mosquitoes were resistant to DDT, while fully susceptible to deltamethrin and malathion. The knock-down values (KDT $10, \mathrm{KDT}_{50}$ and $\mathrm{KDT}_{99}$ ) obtained for DDT displayed straight line in log-dose-probit analysis and follow linear regression model. The KRR ${ }_{99}$ for DDT was 4.9, which indicated a 4.9 folds increase in knock-down resistance to DDT. However, for malathion and deltamethrin, the KRR ${ }_{99}$ values were 1.6 and 1.5 respectively suggesting that mosquitoes were knock-down sensitive. None of the mosquito pool was dengue virus positive.
\end{abstract}

Conclusion: St. albopicta showed resistance to DDT and reduced sensitivity to deltamethrin and malathion. This data on insecticide resistance could help public health authorities in India to design more effective vector control measures. More dengue vector specimens need to be scanned to identify the potential dengue vector.

Keywords: St. albopicta, Dengue, Northeast India, Insecticide resistance, VectorTest ${ }^{\mathrm{TM}}$ assay

\section{Background}

Dengue fever is one of the major mosquito-borne viral infections in tropical and sub-tropical regions. In recent years, dengue transmission has increased predominantly in urban, semi-urban settings and has even extended to the rural settings and become a major public health concern at international level. The World Health Organisation (WHO) has estimated that over 2.5 billion people corresponding to over $40 \%$ of the world's population are

\footnotetext{
* Correspondence: sunildhiman81@gmail.com

Defence Research Laboratory, Tezpur Assam-784 001, India
}

now at dengue risk [1]. In Southeast-Asian countries, the dengue outbreaks usually occur during the rainy season and are supported by Stegomyia aegypti mosquitoes in urban settings, while Stegomyia albopicta in the rural areas [2-4]. St. albopicta (previously Aedes (Stg) albopictus), popularly known as "Asian tiger mosquito", feeds on humans in gardens, parks and bushes around human dwellings in the daytime and is very common in most of the Asian countries $[5,6]$. Since there is no specific vaccine and treatment available for dengue, the control 
mainly turns to the surveillance and control of vectors to reduce transmission in the endemic settings [7-10].

Dengue vector control strategies largely depend upon the use of larvicides in the breeding sites to target the vectors at larval level and space sprays to target the adult infective stages of the potential known dengue vectors. The space spraying is generally used during the outbreak situations, but may be used to target both larvae and adult stages at a time in an integrated approach. Inspite of tremendous anti-vector efforts, the vector control interventions are sometimes threatened due to the development and spread of insecticide resistance among the vector species $[10,11]$. Many recent studies have demonstrated either the development of resistance or decrease in the susceptibility to synthetic insecticides in St. albopicta and St. aegypti mosquitoes in many endemic countries [10-12], however, such data, especially on St. albopicta is very limited in India and only a few systematic studies have been carried out to establish its susceptibility status $[13,14]$. In India, recently dengue has dramatically spread to many areas where it was not reported earlier, owing to which there is urgent need to determine the susceptibility status of the prevalent dengue vector to the commonly used insecticides in the control programme. Further, active surveillance of infected mosquitoes to identify the vector may also be valuable in defining spatial and temporal risk of acquiring dengue infection in an area of interest. Currently, we have examined the distribution and insecticide susceptibility of both larval and adult St. albopicta in Tezpur military area of Northeast India. Tezpur military area is strategically important because it serves as a transit station for the Indian troops moving toward the forward areas along the Indo-China border. Keeping in view the fact that in 2013, the region has reported around 5,000 confirmed dengue cases, the present study was undertaken to establish the insecticide resistance status against the commonly used insectides and to identify the prevalent dengue vector in order to implement effective and sustainable arbovirus vector control measures in the region.

\section{Methods}

\section{Stegomyia mosquito collection}

Stegomyia mosquito aquatic stages (larvae and pupae) were collected in twenty one locations in the military station area ( $26^{\circ} 39^{\prime} 10.2^{\prime \prime}$ to $\left.\mathrm{E} 92^{\circ} 47^{\prime} 33.1^{\prime \prime}\right)$. The immature stages $\left(\mathrm{F}_{0}\right.$-generation) were collected from different types of breeding localities such as, storage tanks, tyres, tree holes, cut bamboos, construction sites, flower pots, plastic cups and drains available in the study area. For each sampling site, larvae or pupae from all the accessible breeding places were collected and transferred to the laboratory. Stegomyia mosquitoes are regularly maintained at the insectary of Defence Research Laboratory,
Tezpur at controlled conditions (temperature- $25^{\circ} \mathrm{C}+/-$ $2^{\circ} \mathrm{C}$; relative humidity $\left.80 \%+/-10 \%\right)$ and the females are fed on rabbit blood to complete their gonotrophic cycle. The wild collected mosquitoes were reared to the adult stages in the laboratory and kept in an isolated room to prevent the inbreeding after identification to species level. The F1-generation of the collected mosquitoes was used for larval and adult bioassays. Further, the laboratory strain (LS) of the mosquitoes was used as reference strain to compare with the wild strain (WS).

\section{Larval bioassay}

Susceptibility of larvae to the temephos (90.7\% pure; Heranba Industries Ltd., Mumbai) was estimated using standard WHO bioassays [15]. The stock solution of $1 \mathrm{ppm}$ and dilutions was prepared in $95 \%$ ethanol and stored at $+4^{\circ} \mathrm{C}$ for use in the experiments. Bioassays were conducted using 20-25 third instar larvae (both WS and LS) in plastic cups filled with required concentration of insecticide solution and millipore water (Milli-Q, MA, USA) at room temperature $\left(25^{\circ} \mathrm{C} \pm 2^{\circ} \mathrm{C}\right)$. Four different concentrations $(0.001,0.005,0.01$ and $0.05 \mathrm{ppm})$ were used and each experiment was replicated at least three times. Each bioassay was accompanied by a control test to which only $95 \%$ ethanol was added in equal concentration. Corrected mortality was calculated after $24 \mathrm{~h}$ and the larvae were considered dead if they did not show any movement when induced with a glass rod.

\section{Adult susceptibility assay}

The adult insecticide susceptibility assay was performed following standard WHO protocol and involved tarsal contact exposure to insecticide impregnated papers in the standard susceptibility test kit $[16,17]$. Susceptibility to $4 \%$ DDT, $0.05 \%$ deltamethrin and $5 \%$ malathion was assessed using insecticide pre-impregnated papers obtained from Universiti Sains Malaysia, Malaysia. Adult unfed females (3-5 days old) in the batches of 10-15 were exposed to the insecticide impregnated papers in the WHO tubes for $1 \mathrm{~h}$ and cumulative knock-down was recorded every 5 minutes. The surviving mosquitoes were transferred to the WHO holding tubes and fed on 5\% sucrose solution. Mortality was scored after $24 \mathrm{~h}$ to grade the sensitivity status as per WHO recommendation [16].

\section{Dengue antigen assay}

The adult Stegomyia mosquitoes flying or resting inside the barracks, washrooms and other places of high density, were collected to detect the dengue virus using VectorTest $^{\text {tax }}$ (Vector Test System Inc., CA) dengue antigen assay. The VectorTest ${ }^{\mathrm{tm}}$ assay is a highly specific and rapid immunochromatographic assay for qualitative detection of any of the four serotypes of dengue 
virus (DENV 1-4) in the infected mosquito [18]. The assays were performed following standard manufacturer's instructions.

\section{Data analysis}

Mortality obtained was corrected using Schneider-Orelli's formula [19] and interpreted following WHO recommendation. Data from larval bioassays were analyzed using Line Log-Dose Probit software. Chi-square $\left(x^{2}\right)$ test was used to estimate the goodness of fit, whereas linear regression $\left(\mathrm{r}^{2}\right)$ was used to evaluate if the data deviate from the linearity. Lethal concentrations $\left(\mathrm{LC}_{10}\right.$, $\mathrm{LC}_{50}$ and $\mathrm{LC}_{99}$ ) along with the slope were estimated at 95\% confidence intervals (CIs). The resistance ratios $\left(R R_{10}, R_{50}\right.$ and $\left.R R_{99}\right)$ were calculated by dividing the $\mathrm{LC}_{10}, \mathrm{LC}_{50}$ and $\mathrm{LC}_{99}$ values of WS with the similar values of LS (reference strain). A $R_{99}$ of $<2$ corresponded to susceptible, whereas $\geq 2$ was considered corresponding to the resistance. Knock-down time (KDT ${ }_{10}, \mathrm{KDT}_{50}$ and $\mathrm{KDT}_{99}$ ) and their 95\% CIs were determined and used to calculate the knock-down resistance ratios (KRRs) $\left(\mathrm{KRR}_{10}, \mathrm{KRR}_{50}\right.$ and $\left.\mathrm{KRR}_{99}\right)$ by dividing the $\mathrm{KDT}_{10}, \mathrm{KDT}_{50}$ and $\mathrm{KDT}_{99}$ values of WS with the similar values of LS. A $K R_{99}$ of $<2$ implied susceptible, while $\geq 2$ was considered implying knock-down resistance in WS St. albopicta.

\section{Results}

The baseline $\mathrm{LC}_{10}, \mathrm{LC}_{50}$ and $\mathrm{LC}_{99}$ value obtained in the LS larval bioassay exhibited a straight-line relationship between the insecticide log dose and probit mortality $\left(x^{2}=1.28 ; p=0.6\right)$. Linear regression showed that there was no deviation among the LC data from linearity $\left(\mathrm{r}^{2}=0.70\right)$ (Table 1$)$. Similarly the $\mathrm{LC}_{10}, \mathrm{LC}_{50}$ and $\mathrm{LC}_{99}$ values observed in the WS also displayed in straight line probit relationship $\left(x^{2}=2.34 ; p=0.3\right)$ and the data did not deviate from linearity $\left(r^{2}=0.61\right)$. The $R R$ values ranged from 1.4 (for $R R_{99}$ ) to 1.7 (for $R R_{50}$ ), which suggest that the tested populations were susceptible to temephos (Table 1).

The results of insecticide susceptibility bio-assay for adult St. albopicta have been shown in Table 2. Corrected

Table 1 Susceptibility of St. albopicta larvae to temephos

\begin{tabular}{lll}
\hline Values & WS (N = 293) & LS (N = 300) \\
\hline $\mathrm{LC}_{10}(95 \% \mathrm{Cl})$ & $0.0006(0.0003-0.001)$ & $0.0004(0-0.001)$ \\
$\mathrm{LC}_{50}(95 \% \mathrm{Cl})$ & $0.0035(0.0026-0.0046)$ & $0.0021(0.0004-0.0037)$ \\
$\mathrm{LC}_{99}(95 \% \mathrm{Cl})$ & $0.081(0.045-0.20)$ & $0.056(0.026-0.60)$ \\
$X^{2}(\mathrm{p})$ & $2.34(0.3)$ & $1.28(0.6)$ \\
$\mathrm{r}^{2}(\mathrm{p})$ & $0.61(0.1)$ & $0.70(0.2)$ \\
$\mathrm{RR}_{10} / \mathrm{RR}_{50} / \mathrm{RR}_{99}$ & $1.5 / 1.7 / 1.4$ & \\
\hline
\end{tabular}

WS- wild strain; LS - lab strain; LC - lethal concentration (ppm); RR- resistance ratio. mortality (CM) obtained for DDT was 57.4 indicating that the mosquitoes were resistant to DDT. However, CM for deltamethrin and malathion was 98.8 and 98.6 respectively, which suggested that St. albopicta adult stage was fully susceptible to both of these insecticides. The knockdown values $\left(\mathrm{KDT}_{10}, \mathrm{KDT}_{50}\right.$ and $\left.\mathrm{KDT}_{99}\right)$ obtained for DDT displayed straight line in log-dose-probit analysis and follow a linear regression model for knockdown with time $(\mathrm{p}=0.9)$. The $\mathrm{KRR}_{99}$ for DDT was 4.9 , indicating that there was a 4.9 fold increase in knock-down resistance to DDT. However, in case of malathion and deltamethrin, the $\mathrm{KRR}_{99}$ values were 1.6 and 1.5 respectively suggesting that mosquitoes were knock-down sensitive. In case of malathion and deltamethrin, the $\mathrm{KRR}_{10}$ values were 4.6 and 4.2 respectively and decreased thereafter for $\mathrm{KRR}_{50}$ and $K R R_{99}$, indicating that mosquitoes had developed a certain level of tolerance to these insecticides which enabled them to survive the knock-down effect up to some time.

Altogether 16 pools of female $(\mathrm{N}=393)$ and 3 pools $(\mathrm{N}=31)$ of adult male St. albopicta were tested for the presence of dengue virus in dipstick based strip assay but none of the pool was found positive for dengue virus.

\section{Discussion}

During the last five decades, use of insecticides in agriculture as well as in public health has led to the development of resistance in mosquito vectors in many endemic countries $[10,11,14,20]$. In the present study, the larvae of the potential dengue vector St. albopicta were completely susceptible to the temephos and the LC values followed a normal linear distribution. The RR values at $\mathrm{LC}_{50}$ or $\mathrm{LC}_{99}$ were lower than 2 , which are not considered biologically significant for resistance. The difference in $\mathrm{RR}$ values at $\mathrm{LC}_{50}$ or $\mathrm{LC}_{99}$ might be due to natural variations in toxicity ratios rather than to resistance selection [21]. Temephos is an organophosphate compound widely used as larvicide to control the vector mosquitoes. Recently, resistance to this insecticide has been reported in many Asian countries [12,22]. However, some studies have indicated that temephos is completely susceptible against wild Ae. albopictus and Ae. aegypti mosquitoes [10,14].

Adult bioassay results showed that the test mosquito species has developed resistance to DDT. Resistance to DDT might have been developed over time due to prolonged use in public health programmes for many years now. DDT resistance has been widely reported in Aedes mosquitoes worldwide [23,24], including India [14], however, the underlying mechanism still remains unclear. Although, the $k d r$ mutation of voltage-gated sodium channel has been reported to confer resistance to DDT, but detoxifying enzyme activity can also play a vital role in the 
Table 2 Mortality and knock-down in St. albopicta against the insecticides; 4\% DDT, 0.05\% deltamethrin (DM) and 5\% malathion (MA)

\begin{tabular}{|c|c|c|c|c|c|c|c|}
\hline Insecticide (N) & $\mathrm{CM}$ & $\begin{array}{c}\mathrm{KDT}_{10} \\
(95 \% \mathrm{Cl})\end{array}$ & $\begin{array}{c}\mathrm{KDT}_{50} \\
(95 \% \mathrm{Cl})\end{array}$ & $\begin{array}{c}\mathrm{KDT}_{99} \\
(95 \% \mathrm{Cl})\end{array}$ & $x^{2}$ & $p$ & $\mathrm{KRR}_{10 / 50 / 99}$ \\
\hline \multirow[t]{2}{*}{ DDT (143) } & 57.4 & 27.0 & 88.5 & 763.7 & 4.1 & 0.9 & $1.9 / 1.2 / 4.9$ \\
\hline & & $(21.2-31.4)$ & (69.9-137.8) & $(359.3-3429.4)$ & & & \\
\hline \multirow[t]{2}{*}{ DM (182) } & 98.8 & 3.8 & 9.4 & 48.0 & 16.8 & 0.002 & $4.2 / 2.1 / 1.5$ \\
\hline & & $(1.6-4.4)$ & $(6.0-12.3)$ & $(42.8-131.4)$ & & & \\
\hline MA (174) & 98.6 & 28.4 & 74.1 & 422.0 & 94.3 & 0.0 & $4.6 / 3.2 / 1.6$ \\
\hline
\end{tabular}

development of DDT resistance [23]. KDT values in the current study indicated that there was a high level (4.9 folds) of knock-down resistance in St. albopicta. Knockdown resistance is one of the most important forms of resistance related to the elevated detoxifying enzyme level in the mosquitoes.

The present study indicated complete susceptibility of St. albopicta mosquitoes to malathion and deltamethrin as the mortality obtained was above 98 percent. Malathion has been used mostly in fogging operations during the mosquito-borne diseases outbreaks and its activity is closely related to the acetylcholinesterase level of target mosquitoes. Pyrethroid resistance has been reported widely $[11,23,24]$, and is associated with an altered amino acid sequence of the sodium channel and also through the elevated activity of detoxification genes. The $\mathrm{KRR}_{99}$ values of malathion and deltamethrin were below 2 , suggesting that St. albopicta mosquitoes were knockdown sensitive. The KDT values obtained in both these insecticides did not display a normal distribution pattern. KDT values have been found to be associated with the level of use of insecticide [20]. No evidence of cross resistance to DDT and deltamethrin was observed in the current study, suggesting the involvement of metabolic resistance rather than a mutation in the sodium channel gene. In India, the data on insecticide resistance status of dengue vectors is scanty and therefore might be a limiting factor for the success in various control efforts. The intervention programmes are often recommended on the basis of evaluation at laboratory level but ignore the evaluation using wild mosquitoes from the field. Insecticide resistance is a dynamic phenomenon and even closely located populations could reflect variation in the insecticide susceptibility level.

Although none of the pool could be found positive for dengue virus, but this study is the first effort aimed at confirming identification of dengue vectors in Northeastern India. Many recent studies have detected the dengue virus in rural and urban settings of India and identified St. albopicta as a potential dengue vector $[3,4]$. A recent report on detecting dengue vector using Vector Test ${ }^{\mathrm{TM}}$ assay has suggested that the assay is highly specific and does not cross-react with other similar viruses [18]. The present study, probably due to the small sample size of St. albopicta mosquitoes used for detecting dengue virus was not able to identify dengue vectors but the present result cannot be generalised to the other areas where dengue outbreaks have been reported recently. Similar investigations using large numbers of possible dengue vectors in different endemic areas are urgently needed to identify potential dengue vectors.

\section{Conclusion}

Wild collected St. albopicta was resistant to DDT and susceptible to temephos, malathion and deltamethrin. There was high level of knock-down resistance developed to DDT. Since dengue cases are rising annually, it is recommended that regular resistance surveillance should first be focused in areas of high dengue fever transmission in order to facilitate selection of insecticides with relatively higher assurance to minimise dengue infections. The identification of dengue vectors using easy and rapid assays and large sample size from different field areas could be useful to target the interventions.

\section{Competing interest}

The authors declare that they have no competing interest.

\section{Authors' contributions}

SD and BR designed the experiments and performed the field study. SD, BR and $K Y$ performed the laboratory studies. SD and KY analysed the data and performed stastical analysis. SD and BR prepared the manuscript. IB and W critically reviewed the manuscript. All the authors have read and approved the final manuscript.

\section{Acknowledgement}

The authors are thankful to health assistants of $118 \mathrm{FHO}, 4$ Corps $\mathrm{HQ}$ and unit Commanding Officers for their help rendered during the study.

Received: 27 February 2014 Accepted: 26 June 2014 Published: 1 July 2014 


\section{References}

1. WHO: Dengue and severe dengue.. Facts sheet No 117. 2013. http://www.who. int/mediacentre/factsheets/fs117/en/ (assessed on 24 Dec 2013).

2. Chow VTK, Chan YC, Yong R, Lee KM, Lim LK, Chung YK, Lamphua SG, Tan BT: Monitoring of dengue viruses in field-caught Aedes aegypti and Aedes albopictus mosquitoes by a type-specific polymerase chain reaction and cycle sequencing. Am J Trop Med Hyg 1998, 58(5):578-586.

3. Kumari R, Kumar K, Chauhan LS: First dengue virus detection in Aedes albopictus from Delhi, India: its breeding ecology and role in dengue transmission. Trop Med International Hlth 2011, 16(8):949-954.

4. Tewari SC, Thenmozhi V, Katholi CR, Manavalan R, Munirathinam A, Gajanana A: Dengue vector prevalence and virus infection in a rural area in south India. Trop Med International HIth 2004, 9(4):499-507.

5. Reinert JF, Harbach RE, Kitching IJ: Phylogeny and cliassification of Aedini (Diptera: Culicidae): based on morphological characters of all life stages. Zool J Linnean Soc 2004, 142:289-368.

6. Higa Y: Dengue vectors and their spatial distribution. Trop Med Health 2011, 39(4):17-27.

7. Bangs MJ, Pudiantari R, Gionar YR: Persistence of dengue viral RNA in dried Aedes aegypti (Diptera: Culicidae) exposed to natural tropical conditions. J Med Entomol 2007, 44:163-167.

8. Bangs MJ, Tan R, Listiyaningsih E, Kay BH, Porter KR: Detection of dengue viral RNA in Aedes aegypti (Diptera: Culicidae) exposed to sticky lures using reverse-transcriptase polymerase chain reaction. J Med Entomol 2001, 38:721-724.

9. Tan CH, Wong PSJ, Li MZI, Vythilingam I, Ng LC: Evaluation of the dengue NS1 Ag Strip ${ }^{\circledast}$ for detection of dengue virus antigen in Aedes aegypti (Diptera: Culicidae). Vector-born Zoon Dis 2011, 1(6):789-792.

10. Kamgang B, Marcombe S, Chandre F, Nchoutpouen E, Nwane P, Etang J, Corbel V, Paupy C: Insecticide susceptibility of Aedes aegypti and Aedes albopictus in Central Africa. Parasit Vectors 2011, 4:79.

11. Dusfour I, Thalmensy V, Gaborit P, Issaly J, Carinci R, Girod R: Multiple insecticide resistance in Aedes aegypti (Diptera: Culicidae) populations compromises the effectiveness of dengue vector control in French Guiana. Mem Inst Oswaldo Cruz 2011, 106(3):346-352.

12. Khan HAA, Akram W, Shehzad K, Shaalan EA: First report of field evolved resistance to agrochemicals in dengue mosquito, Aedes albopictus (Diptera: Culicidae), from Pakistan. Parasit Vectors 2011, 4:146.

13. Selvi S, Edah MA, Nazni WA, Lee HL, Tyagi BK, Sofian-Azirun M, Azahari AH: Insecticide susceptibility and resistance development in malathion selected Aedes albopictus (Skuse). Trop Biomed 2010, 27(3):534-550.

14. Singh RK, Dhiman RC, Mittal PK, Dua VK: Susceptibility status of dengue vectors against various insecticides in Koderma (Jharkhand), India. J Vector Borne Dis 2011, 48:16-118.

15. WHO: Guidelines for laboratory and field testing of mosquito larvicides. In WHO/CDS/WHOPES/GCDPP.13. Geneva, Switzerland: World Health Organization; 2005

16. WHO: Test procedures for insecticide resistance monitoring in malaria vectors, bio-efficacy and persistence of insecticide on treated surfaces. In WHO/CDS/CPC/MAL/98.12. Geneva, Switzerland: World Health Organization; 1998.

17. WHO: Guidelines for testing mosquito adulticides for indoor residual spraying and treatment of mosquito nets. In WHO/CDS/NTD/WHOPES/ GCDPP/3. Geneva, Switzerland: World Health Organization; 2006.

18. Wanja E, Parker ZF, Odusami O, Rowland T, Dave K, Dave S, Turell MJ: Immuno-chromatographic wicking assay for the rapid detection of dengue viral antigens in mosquitoes (Diptera: culicidae). J Med Entomol 2014, 51(1):220-225.

19. Puntener W: Manual for field trials in plant protection. 2nd edition. Ciba-Geigy Limited: Agricultural Division; 1981

20. Dhiman S, Rabha B, Talukdar PK, Das NG, Yadav K, Baruah I, Singh L, Veer V: DDT \& deltamethrin resistance status of known Japanese encephalitis vectors in Assam, India. Indian J Med Res 2013, 138:988-994.

21. Araujo AP, Diniz DFA, Helvecio E, de Barros RA, de Oliveira CMF, Ayres CFJ: The susceptibility of Aedes aegypti populations displaying temephos resistance to Bacillus thuringiensis israelensis: a basis for management. Parasit Vectors 2013, 6:297.

22. Chen CD, Nazni WA, Lee HL, Sofian-Azirun M: Susceptibility of Aedes aegypti and Aedes albopictus to temephos in four study sites in Kuala Lumpur City Center and Selangor State, Malaysia. Trop Biomed 2005, 22:207-216.
23. Chareonviriyaphap T, Bangs MJ, Suwonkerd W, Kongmee M, Corbel V, Ngoen-Kla R: Review of insecticide resistance and behavioral avoidance of vectors of human diseases in Thailand. Parasit Vectors 2013, 6:280.

24. Karunaratne SHPP, Weeraratne TC, Perera MDB, Surendran SN: Insecticide resistance and, efficacy of space spraying and larviciding in the control of dengue vectors Aedes aegypti and Aedes albopictus in Sri Lanka. Pest Biochem Physiol 2013, 107(1):98-105.

doi:10.1186/1756-3305-7-295

Cite this article as: Dhiman et al:: Insecticide susceptibility and dengue vector status of wild Stegomyia albopicta in a strategically important area of Assam, India. Parasites \& Vectors 2014 7:295.

\section{Submit your next manuscript to BioMed Central and take full advantage of:}

- Convenient online submission

- Thorough peer review

- No space constraints or color figure charges

- Immediate publication on acceptance

- Inclusion in PubMed, CAS, Scopus and Google Scholar

- Research which is freely available for redistribution

Submit your manuscript at www.biomedcentral.com/submit
C Biomed Central 